\title{
A Deep Neural Network for Pixel-Wise Classification of Titanium Microstructure
}

\author{
Sirodom Mongkhonthanaphon and Yachai Limpiyakorn
}

\begin{abstract}
In recent decades, deep learning has been widely used for automatically classifying images with high accuracy. Semantic segmentation is a type of deep learning that focuses on classifying every pixel into classes. In Metallurgy domain, Titanium and its alloys are considered an ideal biomaterial that suit for implant material. In this research, deep learning is thus introduced as a means to automate the pixel-wise classification of Titanium microstructure in order to reduce the time and uncertainties during human inspection for quality control. The method applies a semantic segmentation technique, fully convolutional neural network, implemented with the U-net architecture. The proposed approach has investigated the integration of Fine-tuning to the U-net architecture for improving the model performance. The dataset of Ti-6A1-4V microstructure images is augmented using elastic deformations. The results report slightly increase of accuracy, while the training time is much faster than training from scratch.
\end{abstract}

Index Terms-Fine-tuning, fully convolutional neural network, semantic segmentation, titanium microstructure.

\section{INTRODUCTION}

For adulthood humans, there are 206 bones of which the shape is individual and complex. Some of them also serve movement functions that require specific dimensions. These requirements and constraints lead to the pain points for implant material designers and the manufacturing process to produce complex part geometries. In recent decades, titanium and its alloys have been the solution widely used as implant material. Titanium-based alloys are considered an ideal biomaterial, of which the properties exhibit high biocompatibility, high mechanical strength, high fatigue resistance, good wear resistance and low density [1]. The most common industrial grade for implant is the Ti-6Al-4V (6\% Aluminium, 4\% Vanadium) titanium alloy. The addition of alloying elements aims to enhance its phase stabilization, lowering the temperature of the transformation from Alpha phase to Beta phase [2]. Aluminium tends to stabilize the Alpha phase, while Vanadium tends to stabilize the Beta phase. It is crucial to make dual phase titanium alloys for high-taken load implant material. Although single Alpha phase has excellent strength and oxidation resistance, it has low capability of absorbing deformation energy, whereas dual Alpha-Beta phase has much higher capability of

Manuscript received February 26, 2019; revised October 11, 2019

The authors are with the Department of Computer Engineering, Chulalongkorn University, Bangkok 10330, Thailand (e-mail: seerowdom@gmail.com, yachai.1@chula.ac.th). absorbing deformation energy.

In industry, among others, selective laser melting (SLM) seems to suit for producing implant material, with the ability to make complex shapes, reduce fabrication time and material waste from the producing process. However, as-built state titanium parts produced by SLM are unable to achieve the great performance. Due to their single Alpha phase microstructure, toughness and ductility are not high enough to be used as high-taken load. Improving the toughness and ductility of titanium-based alloys, heat treatment is required to perform dual Alpha-Beta phase. Heat treatment is the post-process that changes microstructural morphology to improve some mechanical behaviors. Ter Harr and Becker [3] experimented the heat treatment process on Ti-6Al-4V produced by SLM method. The result showed a significant increase of $\%$ elongation for the samples that have been heated above $750^{\circ} \mathrm{C}$ (dissolution temperature). An as-built state of Ti-6Al-4V consists only the Alpha phase. The dissolution temperature is the temperature that the Alpha phase is about to start dissolve into Beta phase. The other matters are time and the method of cooling that can affect the microstructure morphology. Once the heat treatment has been completed, microstructure needs be visualized to ensure that the heat treatment process was done properly.

Ti-6Al-4V is the commercial grade for biochemical implant. During the inspection process for quality control, domain expertise is required for identifying the phase transformation. The domain experts are those metallurgy engineers who study titanium microstructure phase transformation. Mostly of microstructure visualization is manually performed by metallurgists including the task of phase classification. In this work, deep learning is introduced as a means to automate the pixel-wise classification of Alpha or Beta phase contained in the Ti-6Al-4V microstructure, that would result in reducing the time and uncertainties during human inspection for quality control.

\section{DEEP LEARNING}

Deep Neural Networks is the technique opted for Pixel-Wise Classification of Titanium Microstructure in this work. Technically, Deep Learning is a subset of Machine Learning. It makes use of deep neural networks or use of multiple layers of neurons to progressively extract features from raw input. By mapping the consistency of features by feed-forward process and optimizing the regularize loss function from back propagation process, the deep learning networks are expected to recognize the patterns hidden in a supervised dataset and to automatically create feature maps to extract features from the dataset. The model then uses the recognized knowledge from the pattern learning to perform 
prediction on the new or unseen dataset.

In image processing, the deep learning algorithm like Convolutional Neural Networks (CNNs) as shown in Fig. 1 (source: www.kdnuggets.com), the shallower layers may identify more generic information features such as edges and lines, while the deeper layers may identify more specific information features such as meaningful specific features in the dataset. Deep learning architectures are often empirically constructed layer by layer, and there are such hyperparameters in the networks that must be tuned during the learning process to adjust the networks. The hyperparameters tuning process is directly conducted contributing to model accuracy and training time. Starting from the initial of convolution layers in CNN, filters are initialized by randomly Gaussian distribution. During the training of the networks, these filters learn more patterns hidden in the dataset as the network gets deeper. These filters represent the operations to extract feature maps contained in the networks. While pooling layers are used to sub-sampling the feature maps dimension after being convoluted and to represent the new smaller feature maps. These two types of layers are alternately constructed in the convolution base of the CNN networks before the last classification part that will perform classifying. The classification layer is generally the fully connected neural networks that use the input as flattened feature maps from the last layer of convolution base. There are some of the techniques that are often applied to prevent the deep learning networks from overfitting the dataset by adding the regularization in the feed forward learning process. One of the famous regularization techniques, so called dropout layer, is the means to randomly drop some nodes of the individual neural network nodes. After having applied dropout, the networks nodes are reduced by the fraction of dropout parameters and the edges from the nodes that are removed are also disconnected. These techniques could affect the nodes that are dominant and could be weighted more than the other nodes. Dropout technique can also be used in the beginning input of the networks to perform noises in the input images that could make the deep neural network performance more robust to the uncertainty input images. Another famous technique, so called batch normalization, is often used to normalize the output parameters before the activation function by subtracting the batch mean and dividing by the batch standard deviation. For the CNN input, the structure must be defined with certain dimensions. Zero-padding could be performed to help prevent dimensionality loss.

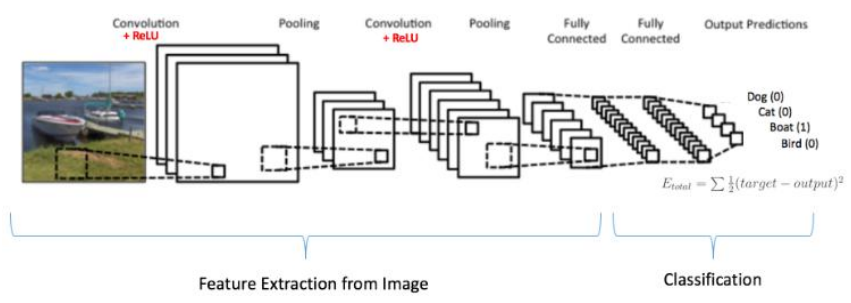

Fig. 1. Architecture of Convolutional Neural Network as example of deep learning technique.

\section{RELATED WORK}

Computer vision is a challenging field studying on how computer could extract the feature in the images processing with pixel input of images generally represented by digits.
Every pixel in the images could be related to the meaningful insight to fulfill the on-purpose task of computer vision. One of the computer vision studies intriguing many researchers is semantic segmentation that uses every pixel in the image to classify the classes. The benefit of using semantic segmentation is to recognize the insight of images as localization and classification.

Microstructural morphology is regarded as fundamental fingerprint for material properties that can tell the background of the material during inspection process. Assuming that the formation of patterns exists in microstructure, it is very challenging to exploit computer vision to recognize the patterns. Gola et al. [4] proposed steel microstructure classification using machine learning methods. Their dataset consists of steel images taken by optical microscope (OM). All images were categorized into three classes: pearlite, martensite and bainite. The result showed the average test accuracy around $80 \%$ on the authors' test dataset. Azimi et al. [5] applied the method of Gola et al. [4] to their dataset, which categorized into four classes: pearlite, tempered martensite, martensite and bainite. But the result reported only $48.89 \%$ accuracy. Azimi et al. therefore proposed steel microstructure classification by deep learning methods, using fully convolutional neural network (FCNN) [6] as pixel-wise classifier and predicted the result by max-voting scheme. The final result achieved 93.94\% classification accuracy and $67.84 \%$ mean IoU. In addition to the field of material science, image segmentation is widely used in biomedical area. Ronneberger et al. [7] proposed applying convolutional networks for biomedical image segmentation. The network architecture is called U-Net, and it won the ISBI cell tracking challenge 2015 by a large margin. The U-net architecture is built from FCNN concept to perform image segmentation. In 2018, Iglovikov and Shvets [8] presented the integration of Fine-tuning technique to the U-net architecture. The purpose is to increase the accuracy with the pretrained network that has been previously trained on a large dataset. The work used the VGG11 architecture as encoding contracting path, and Inria Aerial Image Labeling as the dataset which contains top view urban settlements images, each of which is associated with the ground-truth image labelled as a building or not building class.

Semantic segmentation is a type of deep learning that focuses on classifying every pixel into classes. Semantic segmentation is one of the key problems in the field of computer vision, and it can be applied to a variety of applications including self-driving car, medical diagnosis, virtual reality etc. For example, Chataigner et al. [9] proposed an approach using $\mathrm{CNN}$ as feature extraction and Support Vector Machine (SVM) as semantic segmentation on CITYSCAPE video dataset which is the famous dataset for self-driving car application.

The method of Fully Convolutional Neural Networks is well-known in semantic segmentation. FCNN was proposed by Long et al. in 2015 [6]. Compared to the original CNNs, FCNNs are the model containing upsampling layer instead of fully connected layer. Therefore, FCNNs can deliver the output as the same resolution as the input image. Another difference is Skip Connections that skip the information of the shallower layers to the deeper layers. This method 
improves further precision by fusing the information before the final prediction layer.

\section{Methodology}

\section{A. Architecture}

The U-net architecture can be separated to two divided parts: encoding contracting path and decoding expansive path. In general, the contracting path consists of repeated $3 \times 3$ convolutional operations with a rectified linear unit (ReLU) as the activation function for each convolution and $2 \times 2$ max pooling operations. These repeated operations are regarded as downsampling step, where input images are encoded into small feature maps with higher number of feature channels. The other part is expansive path consisting of repeated upsampling convolutional operations. The output in every step of expansive path are decoded to higher resolution feature map than the previous. According to skip connection technique, expansive path combines the high-resolution feature map from their output and feature map that is concatenated from contracting path together.

Compared to the original CNN model, the contracting path in the U-net architecture can be referred as convolutional base. $\mathrm{CNN}$ is generally composed of the parts of convolutional base and classifier. The convolutional base is repeated convolutional operations and max pooling operations, while the classifier is fully connected layers. As a consequence, the contracting path and convolutional base are replaceable.

Due to limited resources, the pretrained weights of well-known VGG16 that performed on the ImageNet dataset is selected in this work. The pretrained is obtained via Keras author, François Chollet's Github repository. The weights can be downloaded as including classifier part or without classifier part. Here, the pretrained weights without classifier part is adopted. The U-net architecture is modified by replacing contracting path with VGG16-based convolutional neural network, then adjusting the expansive path likewise concatenating feature maps from contracting path.

The architecture is illustrated in Fig. 2. The contracting path is on the left side and the expansive path is on the right side. The operation steps follow along the arrow. The number inside each box indicates the number of channels. The inputs are 3 channels RGB images, while the outputs are 1 channel grayscale images. The concatenate branches show the concatenate copied feature maps path from contracting path to expansive path. The size of feature maps from the concatenated must be the same as in the expansive path, which are increased by $2 \times 2$ upsampling operations to make the merging of two feature maps possible. The fuse of two feature maps can be implied as skip connection via the networks.

\section{B. Fine-Tuning}

Training the model using a small dataset is a common situation, and it is prone to encounter with inaccuracy problem. In case of learning from scratch approach, the model begins naively training with no knowledge. The knowledge that is learned are from the dataset. On the other hand, the fine-tuning approach provides the pretrained weights for initializing the learning knowledge and only adjusts the highly specialized features to make them relevant for the problem at hand. The pretrained weights are usually trained with the large dataset like ImageNet dataset. There are many open sources available on the internet that publicly upload the pretrained weights from the well-known networks such as AlexNet, VGG, ResNet, Xception etc. By using pretrained weights, the model must have the same architecture as the previous model architecture.

In this work, the pretrained weights are loaded into contracting path. All these weights in the layers are frozen, that means they are in the untrainable state. To make its fine-tuning, a few of the top layers of frozen layers need be unfrozen to turn into trainable state, in order to make the model more relevant to the problem at hands.

According to Fig. 2, the pretrained VGG16 weights are loaded and frozen in the contracting path. Once the last $2 \times 2$ Maxpooling has been operated, the following layers are then trainable, allowing the networks to fine-tuning update weights during the new training.

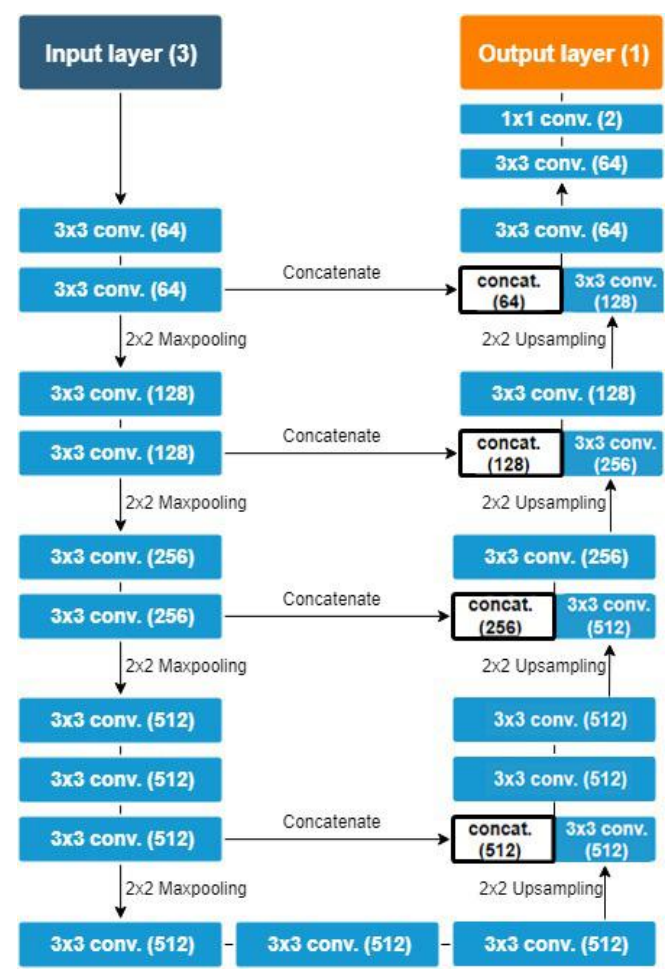

Fig. 2. U-net architecture containing contracting path implemented with VGG16-based convolutional neural network.

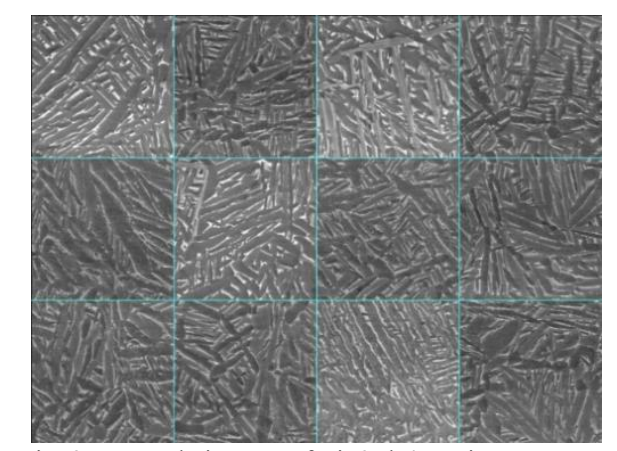

Fig. 3. Example images of Ti-6Al-4V microstructure.

\section{Dataset}

The dataset contains the images of Ti-6Al-4V 
microstructure taken by SEM microscopes. The titanium alloys are produced by SLM method and heated to transform the as-built microstructure into dual-phase (Alpha/Beta) microstructure. The dataset contains 96 images with $512 \times 512$ pixels, examples as shown in Fig. 3. Each image owns its corresponding binary ground-truth image that supervises the classes to the model. The binary ground-truth images, examples as shown in Fig. 4, denote Alpha phase and Beta phase with black color and white color, respectively.

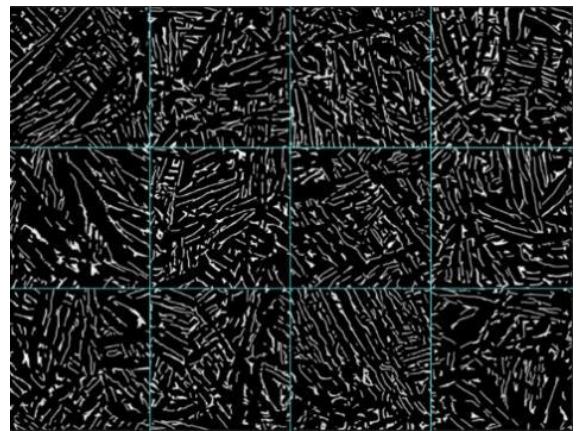

Fig. 4. Images of binary ground truths associated with those in Fig. 3.

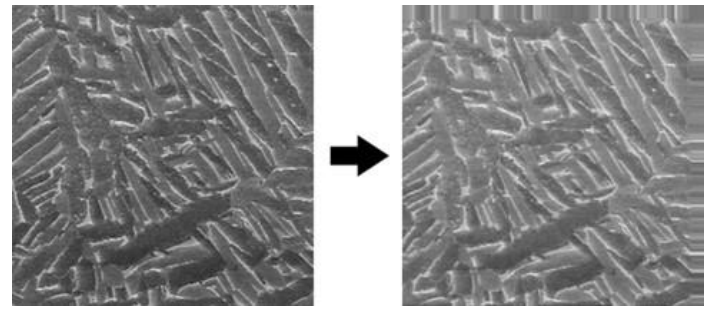

Fig. 5. Example of augmented image of Ti-6Al-4V microstructure (right).

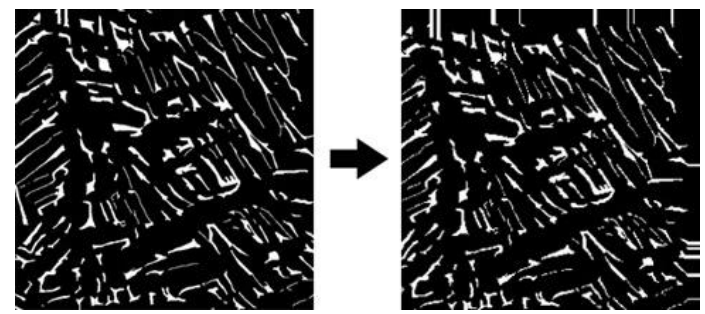

Fig. 6. Example of augmented binary ground-truth (right).

\section{Data Augmentation}

As the dataset is small, data augmentation using elastic deformations [10] is applied for increasing the training data size of 66 to the size of $264(66 \times 4)$ images. Associated with each augmented image, the ground truth image is also created using the same pixel shifting. This step is implemented with Keras using ImageDataGenerator instance, which can set the parameters for elastic deformations in the instance such as:

1) rotation_range is a value in degrees (0-180), a range within which to randomly rotate images. The value is set to rotation range $=0.2$

2) width_shift and height_shift are ranges (as a fraction of total width or height) within which to randomly translate images. The values are set to width_shift_range $=0.05$, height_shift_range $=0.05$.

3) shear_range is for randomly applying shear transformations. The value is set to shear_range $=0.05$

4) zoom_range is for randomly zooming inside images. The value is set to zoom_range $=0.05$

5) horizontal_flip is for randomly flipping half the images horizontally. The value is set to horizontal_flip=True
6) fill_mode is the strategy used for filling in newly created pixels, which can appear after a rotation or a width/height shift. The value is set to fill_mode='nearest

Fig. 5 depicts an example of the augmented image of Ti-6Al-4V microstructure and the associated augmented binary ground-truth as shown in Fig. 6 .

\section{E. Training}

The network produces an output of a 3D tensor with the number of channels equivalent to the number of classes. Since there are only two classes (Alpha and Beta), the ground truth images are thus pre-processed into binary color images by thresholding from grayscale images. Thresholding is manually performed by replacing each pixel in an image with a black pixel if the image intensity is less than some fixed constant, manually chosen by visualizing the histogram color map. In this work, the threshold is set to 128 for the transformation of grayscale ground-truth to binary ground-truth. The images of titanium microstructure are used as input and all binary ground-truth images are used to supervise the network in the output section. For training, Adam optimizer [11] is used with 10-4 learning rate and no weight decay. The loss function is computed by using binary-cross entropy function. To predict the output of the last layer for every pixel, the sigmoid function is applied to score the posterior probability then choosing the class for that pixel with the highest posterior score.

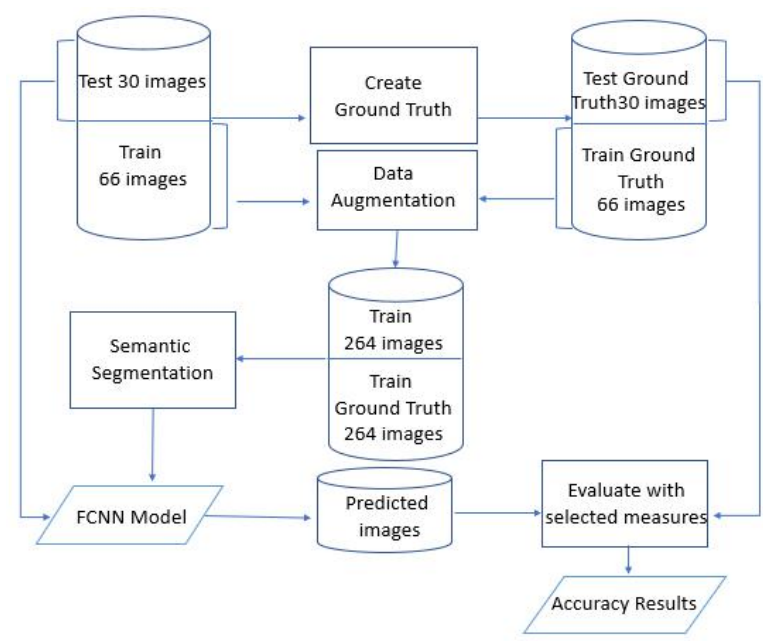

Fig. 7. Training process with data augmentation.

\section{F. Implementation Environments and Tools}

The models were trained under the following environments:

1) Windows 10 Enterprise (64-bit)

2) CPU Intel $\AA$ Processor Core TM i5-6400 $2.70 \mathrm{GHz}$ (4CPUs)

3) RAM 8 GB

4) GPU Nvidia GeForce GTX 1060 (6GB)

The development tools used for training the models include:

1) Python 3.6.4

2) Anaconda3 (64-bit) supporting Jupyter Notebook and Python Libraries e.g. Numpy Pip Wheel.

3) Tensorflow-gpu 1.5.0 as Keras backend

4) Keras 2.1 .4

5) Scikit-Learn 0.14.0 
6) OpenCV-python 3.4 .2

7) Matplotlib 2.1.2

8) Pillow 5.0.0

The dataset of SLM produced Ti-6Al-4V contains totally 96 images that is split into 66 training images and 30 test images. Fig. 7 illustrates the training or learning process with data augmentation for increasing the size of training dataset to 264 images (original $66+$ augmented $66 \times 3$ ). The network was trained with 150 epochs as derived from Fig. 8, accompanied with Fig. 9 that shows the loss function of the model.

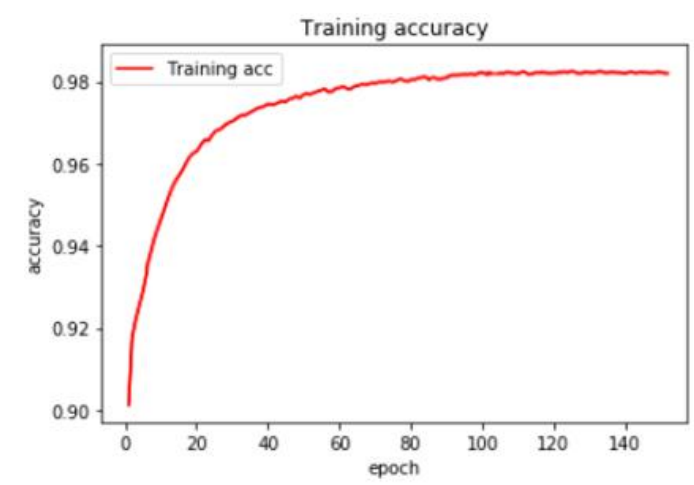

Fig. 8. Visualization of training accuracy.

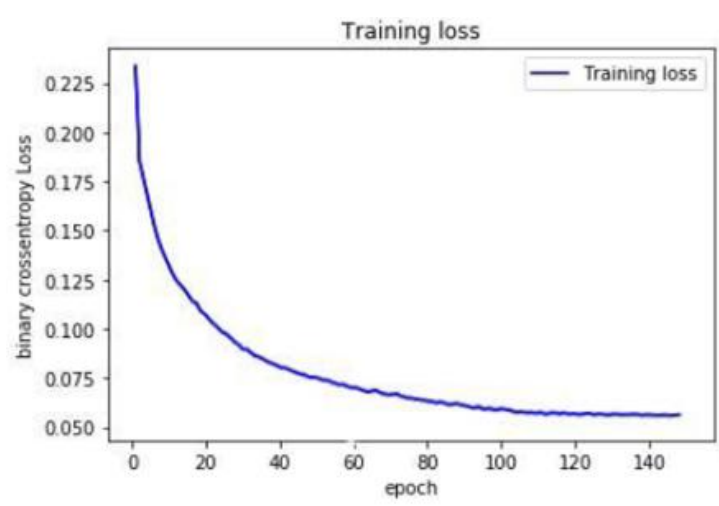

Fig. 9. Visualization of model loss function.

\section{Evaluation MEASURes}

The performance evaluation of the model is measured by four metrics: 1) pixel accuracy, 2) mean accuracy, 3) mean of intersection over union ( $\mathrm{IoU}$ ) and 4) frequency weighted of IoU. These metrics are commonly used for semantic segmentation and scene parsing evaluation.

Given that $n_{i j}$ denotes the number of pixels of class i-th predicted to belong to class $j, n_{c l}$ is number of different classes included in ground truth segmentation, and $t_{i}$ is the total number of pixels of class $\mathrm{i}$-th in ground-truth segmentation.

Pixel accuracy is defined by the proportion of correctly classified pixels as in (1).

$$
\text { pixel accuracy }=\frac{\sum_{i} n_{i i}}{\sum_{i} t_{i}}
$$

Mean accuracy is defined by the proportion of correctly classified pixels averaged over all the classes as in (2).

$$
\text { mean accuracy }=\frac{1}{n_{c l}} \frac{\sum_{i} n_{i i}}{t_{i}}
$$

Mean IoU is defined by the intersection over union (IoU) between the predicted and ground-truth pixels, averaged over all the classes as in (3).

$$
\text { mean IoU }=\frac{1}{n_{c l}} \sum_{i} \frac{n_{i i}}{\left(t_{i}+\sum_{j} n_{j i}-n_{i i}\right)}
$$

Frequency weighted IoU is defined by the IoU weighted by the total pixel ratio of each class as in (4).

$$
\text { frequency weighted IoU }=\sum_{k} \frac{1}{t_{k}} \sum_{i} \frac{t_{i} n_{i i}}{\left(t_{i}+\sum_{j} n_{j i}-n_{i i}\right)}
$$

\section{RESULTS}

The summary of model performance is detailed in Table 1 that reports all the measures on two models (U-net vs. U-net+VGG16) with two strategies: data augmentation, and pretrained weights. In case of applying data augmentation and pretrained weights strategy, the trained network achieves the best pixel accuracy of $93.03 \%$, mean accuracy of $83.07 \%$, mean IoU of $73.17 \%$ and frequency weighted IoU of $87.94 \%$. Adopting data augmentation yields all increasing values of measures for both models (U-net and U-net+VGG16). It is observed that the addition of fine-tuning technique with pre-trained weights from VGG16 to the U-net model that adopts data augmentation yields the best performance measured by the accuracy and training time. The result reveals that using pretrained weights strategy provides much faster converging loss function than the other four scenarios. The training time is only 2 hours on a Nvidia GTX 1060 (6GB), while the training time of the other four strategies are about 6 hours. Fig. 10(c) depicts the predicted image as the output of the model using data augmentation. Fig. 10(d) depicts the predicted image as the output of the model using data augmentation and fine-tuning technique. Compared to Fig. 10(c), Fig. 10(d) is more accurate or more similar to Fig. 10(b).

TABLE I: COMPARISONS OF PERFORMANCE OF U-NET VS. UNET+VGG16

\begin{tabular}{lclllllc}
\hline \multirow{2}{*}{ Model } & \multirow{2}{*}{ Augment } & $\begin{array}{l}\text { Fine } \\
\text { Tune }\end{array}$ & $\begin{array}{l}\text { Pixel } \\
\text { acc. }\end{array}$ & $\begin{array}{l}\text { Mean } \\
\text { acc. }\end{array}$ & $\begin{array}{l}\text { Mean } \\
\text { IoU }\end{array}$ & $\begin{array}{l}\text { FW } \\
\text { IoU }\end{array}$ & $\begin{array}{c}\text { Time } \\
\text { (hrs.) }\end{array}$ \\
\hline Unet & no & no & 80.21 & 60.42 & 55.32 & 80.19 & 6 \\
Unet & yes & no & 81.54 & 62.08 & 60.66 & 83.74 & 6 \\
+VGG & no & no & 83.09 & 67.27 & 65.12 & 82.03 & 6 \\
+VGG & yes & no & 92.67 & 80.39 & 71.30 & 87.24 & 6 \\
+VGG & yes & yes & $\mathbf{9 3 . 0 3}$ & $\mathbf{8 3 . 0 7}$ & $\mathbf{7 3 . 1 7}$ & $\mathbf{8 7 . 9 4}$ & $\mathbf{2}$ \\
\hline \hline
\end{tabular}
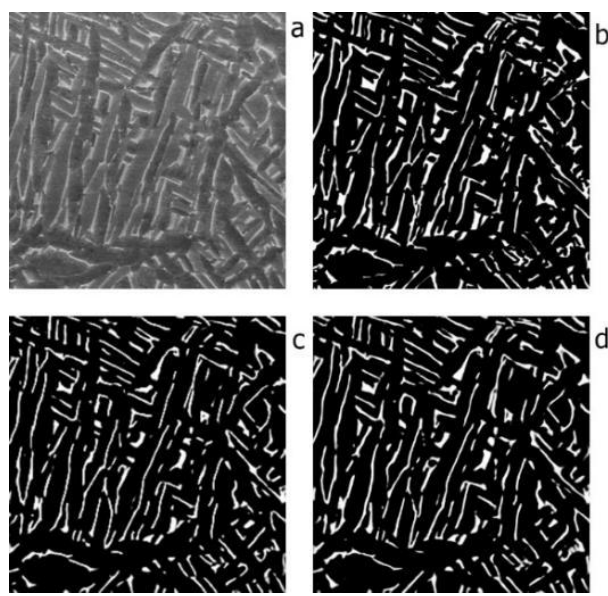

Fig. 10. Example of (a) test image, (b) associated ground-truth image, (c) predicted image using data augmentation, (d) predicted image using data augmentation and fine-tuning. 
In literature, Azimi et al. [5] applied deep learning for classifying phases of steel microstructural namely Martensite, Tempered Martensite, Bainite, and Pearlite. The best performance of their model yielded: Pixel accuracy $93.92 \%$, Mean accuracy $76.70 \%$, Mean IoU $67.84 \%$, and frequency weighted IoU $88.81 \%$.

\section{CONCLUSION}

Due to several suitable properties, titanium alloys have been widely used for implant surgery. To improve the manufacturing process, this paper presents an approach to applying a type of semantic segmentation, Fully Convolutional Neural Networks, for pixel-wise classification. The FCNN classifier is constructed with the U-net architecture to classify the two phases (Alpha or Beta) in Ti-6Al-4V microstructure images. Fine-tuning is introduced as in general, the technique tends to increase accuracy for small datasets by using pretrained weights. The contracting path of U-net is modified to VGG16 architecture to serve the pretrained weights, while most of them are frozen to prevent from new learning but a few of the top layers are unfrozen to open for the new learning, in order to make the model more relevant to the problem at hands. The results show that the performance of the model using fine-tuning is slightly better than no fine-tuning. However, the technique can reduce much training time. Further direction would be changing pretrained weights to the more advanced architecture that is known to achieve better performance in classification such as ResNet and GoogLeNet.

\section{CONFLICT OF INTEREST}

The authors declare no conflict of interest.

\section{AUTHOR CONTRIBUTIONS}

S. M. and Y. L. evenly contribute to the research. All authors had approved the final version.

\section{REFERENCES}

[1] J. Acero, J. Calderon, J. Salmeron, J. Verdaguer, and C. Consejo, "The behavior of titanium as a biomaterial: microscopy study of plates and surrounding tissues in facial osteosynthesis," Journal of Cranio-Maxillofacial Surgery, vol. 27, no. 2, pp. 117-123, 1999.

[2] C. Oldani and A. Dominguez, "Titanium as a biomaterial for implants, recent advances in arthroplasty," Intech Open Science, pp. 150-162, 2012 .

[3] M. Terhaar and T. H. Becker, "Selective laser melting produced Ti-6Al-4V: Post-process heat treatments to achieve superior tensile properties," Journal of Physics, vol. 371, 2018.

[4] J. Gola, D. Britz, T. Staudt, M. Winter, S. A. Schneider, M. Ludovici and F. Mucklich, "Advanced microstructure classification by data mining methods," in Proc. International Conference on ThermoMechanical Processing (TMP), 2016.

[5] S. Azimi, D. Britz, M. Engstler, M. Fritz and F. Mücklich, "Advanced steel microstructural classification by deep learning methods," Nature-Scientific Reports, 2018.

[6] J. Long, E. Shelhamer and T. Darrell, "Fully convolutional networks for semantic segmentation," Computer Vision and Pattern Recognition (CVPR), 2015.

[7] Ronneberger, P. Fischer, and T. Brox, U-Net: Convolutional Networks for Biomedical Image Segmentation, Springer International Publishing, 2015.

[8] V. Iglovikov, and A. Shvets, "TernausNet: U-Net with VGG11 encoder pre-trained on imagenet for image segmentation," Computer Vision and Pattern Recognition (CVPR), 2018.

[9] J. Chataigner, S. Herbin and A. Chan-Hon-Tong, "Pertinence of video for single image deep network," International Journal of Machine Learning and Computing (IJMLC), vol. 7, no. 6, 2017.

[10] M. D. Bloice, C. Stocker, and A. Holzinger, "Augmentor: An image augmentation libray for machine learning," Computer Vision and Pattern Recognition (CVPR), 2017.

[11] P. D. Kingma and L. J. Ba, "Adam: A method for stochastic optimization," in Proc. International Conference on Learning Representations, 2015.

Copyright (C) 2020 by the authors. This is an open access article distributed under the Creative Commons Attribution License which permits unrestricted use, distribution, and reproduction in any medium, provided the original work is properly cited (CC BY 4.0).

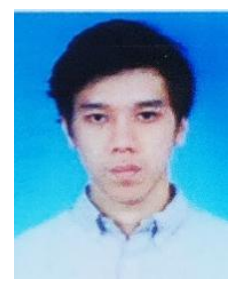

Sirodom Mongkhonthanaphon graduated from the Department of Metallurgical, Faculty of Engineering, Chulalongkorn University, Bangkok, Thailand, in 2016. He earned his master degree in computer engineering from Chulalongkorn University in 2018. His area of research is in machine learning, metallurgical engineering. 